\title{
Effect of arthroscopic acromioplasty on reducing critical shoulder angle: a protocol for a prospective randomized clinical trial
}

\author{
Yi Long, Jingyi Hou, Yiyong Tang, Fangqi Li, Menglei Yu, Congda Zhang and Rui Yang ${ }^{*}$ (D)
}

\begin{abstract}
Background: The critical shoulder angle (CSA), which helps to predict patients who are at risk of rotator cuff tears (RCTs) with large degree and who are susceptible to osteoarthritis with low angle, has been identified as one of the most vital acromial parameters; anterolateral and lateral acromioplasties have been proven to be valid ways to reduce CSA. However, no study has compared the effect of different acromioplasties on the reduction of the large CSA $\left(\geq 33^{\circ}\right)$ clinically. Additionally, either anterolateral or lateral acromioplasty could not precisely correct large CSAs to a favorable range $\left(30-33^{\circ}\right)$ in each patient. Thus, we will propose a novel precise acromioplasty technique for the purpose of reducing CSA accurately and effectively, and compare the effectiveness of different acromioplasties on the reduction of the CSA.

Methods: A total of $60 \mathrm{RCT}$ patients who have indications for arthroscopic rotator cuff repair and with preoperative CSA $\geq 33^{\circ}$ will be recruited in outpatient center of Sun Yat-sen Memorial Hospital. Eligible participants will be randomly allocated to Group A (anterolateral acromioplasty), Group B (lateral acromioplasty) or Group C (precise acromioplasty) via a random, computer-generated number system. Three surgical plans will be made for each participant respectively by one professional surgeon according to the results of randomization allocation. The postoperative CSA will be measured 2 days post-operation. Follow-up will be maintained at 3, 6, and 12 months after surgery including the visual analog scale score, the University of California at Los Angeles score, the Constant Shoulder Score and the American Shoulder and Elbow Surgeon Shoulder Assessment Form. Finally, all outcomes will be assessed by two researchers who are blinded to the recruitment and allocation.

Discussion: This is the first clinical trial to evaluate the impact of different acromioplasties on the reduction of the CSA. Additionally, this study will provide a new precise acromioplasty technique, which is a novel precision and individualized treatment to prevent degenerative RCTs by reducing the CSA.
\end{abstract}

Trial registration: ChiCTR2000032343. Registered on April 26th, 2020.

Keywords: Critical shoulder angle, Acromioplasty, Rotator cuff tears, 3D reconstruction

* Correspondence: yangr@mail.sysu.edu.cn

Department of Orthopedics, Sun Yat-sen Memorial Hospital, Sun Yat-sen

University, 107 Yan Jiang Road West, Guangzhou 510120, Guangdong, China

(c) The Author(s). 2020 Open Access This article is licensed under a Creative Commons Attribution 4.0 International License, which permits use, sharing, adaptation, distribution and reproduction in any medium or format, as long as you give appropriate credit to the original author(s) and the source, provide a link to the Creative Commons licence, and indicate if changes were made. The images or other third party material in this article are included in the article's Creative Commons licence, unless indicated otherwise in a credit line to the material. If material is not included in the article's Creative Commons licence and your intended use is not permitted by statutory regulation or exceeds the permitted use, you will need to obtain permission directly from the copyright holder. To view a copy of this licence, visit http://creativecommons.org/licenses/by/4.0/ The Creative Commons Public Domain Dedication waiver (http://creativecommons.org/publicdomain/zero/1.0/) applies to the data made available in this article, unless otherwise stated in a credit line to the data. 


\section{Background}

Rotator cuff tears (RCTs) is a common shoulder disease in the general adult population $[1,2]$. While the pathogenesis of degenerative RCTs are multi-faceted, the precise mechanisms are still not fully understood [3-5]. The acromial morphology has been identified as one of the important etiologic factors of degenerative RCTs [6-8]. Numerous studies have revealed that a type III acromion, higher acromion index, lower lateral acromion angles, and larger critical shoulder angles (CSAs) are significantly associated with degenerative RCTs [9-11].

The CSA, defined as the angle between the plane of the glenoid fossa and a line connecting the inferolateral point of the acromion with the inferior glenoid margin on standardized anterior radiographs [12], has been identified as one of the most accurate and vital anatomic predictors of the development of degenerative RCTs [13]. Several studies have documented that a CSA of more than $35^{\circ}$ predicts RCTs, whereas a CSA of $30^{\circ}$ or less is associated with glenohumeral osteoarthrtitis (OA) or superior labrum from anterior to posterior lesion (SLAP) [14-19]. Gerber et al. concluded that correcting a large CSA to $33^{\circ}$ or less during arthroscopic rotator cuff repair (RCR) could help patients to achieve superior strength of abduction after the RCTs heal [20]. Similarly, we previously found that a reduction of a large CSA $\left(\geq 33^{\circ}\right)$ to a desired range $\left(30-33^{\circ}\right)$ may prevent $\mathrm{RCTs}$ and OA [21].

Arthroscopic anterolateral or lateral acromioplasty has been proven to change the pathologically increased CSA in cadaveric study [22, 23]. Some authors also reported that the CSA can be reduced significantly by anterolateral or lateral acromioplasty in vivo [20, 24, 25]. Furthermore, Kaiser et al. demonstrated that lateral acromioplasty decreased the CSA more significantly than anterolateral acromioplasty in a three-dimensional analysis [26]. Nevertheless, to our knowledge, no study has yet compared the effect of different arthroscopic acromioplasties on the reduction of the large CSA $\left(\geq 33^{\circ}\right)$ clinically. Besides, although these techniques can greatly reduce CSA, none precisely reduce large CSAs to the favorable range.

The primary goal of the current study is to propose the precise acromioplasty, which is a novel technique for the purpose of reducing the CSA sufficiently and accurately. The second goal is to conduct a prospective randomized clinical trial that will evaluate the effectiveness of different acromioplasties on the reduction of CSA.

\section{Methods}

\section{Study design}

This study is a prospective, single-center, parallel-design randomized controlled trial. This protocol has been developed according to the SPIRIT statement [27]. The trial has been prospectively registered in the Chinese Clinical Trial Registry (ChiCTR2000032343). The outline of the study was given in Fig. 1.

\section{Recruitment and informed consent}

This study will be conducted in Sun Yat-sen Memorial Hospital. Patients with rotator cuff tears (RCTs) who need arthroscopic rotator cuff repair (RCR) will be recruited from the outpatient center of Sun Yat-sen Memorial Hospital. After accomplishing baseline assessment and confirming the eligibility of the patients, the researcher will introduce the study purposes, procedures, possible risks etc. for approximately $45 \mathrm{~min}$, and answer all the questions raised by patients and their families. Informed consent forms will be signed by each patient. After enrollment, participants will be coded with a unique number.

\section{Eligibility and exclusion criteria}

The inclusion criteria are as follows: diagnosis of unilateral degenerative RCTs; pre-operative critical shoulder angle $(\mathrm{CSA}) \geq 33^{\circ}$; required to receive arthroscopic RCR; willing to take part in this study; preoperative three-dimensional computed tomography (3D-CT) and post-operative standard radiographs of shoulder were performed in our institution. The exclusion criteria are: history of shoulder trauma, and concomitant fracture or dislocation of shoulder; previous surgery of the involved shoulder; diagnosis of massive rotator cuff tears; concomitant frozen shoulder, glenohumeral osteoarthrtitis (OA) or other inflammatory arthritis; unable to give informed consent.

\section{Randomization}

After informed consent, eligible participants will receive a unique number. Then, patients will be randomly allocated to either the anterolateral acromioplasty group (Group A), the lateral acromioplasty group (Group B), or precise acromioplasty group (Group C) via a random, computer-generated number system. The treating surgeon will determine surgical plans according to the results of the randomization allocation. Allocation will be based on a 1:1:1 ratio between the three different groups.

\section{Blinding}

In this study, participants will be clearly informed of surgical plans before operation. Besides, the surgeon and assistant will perform acromioplasty along with arthroscopic RCR. Consequently, the patients, treating surgeon and assistant are not blinded to the group allocation. However, the surgeon and assistant will not participate in the pre-operative and post- 


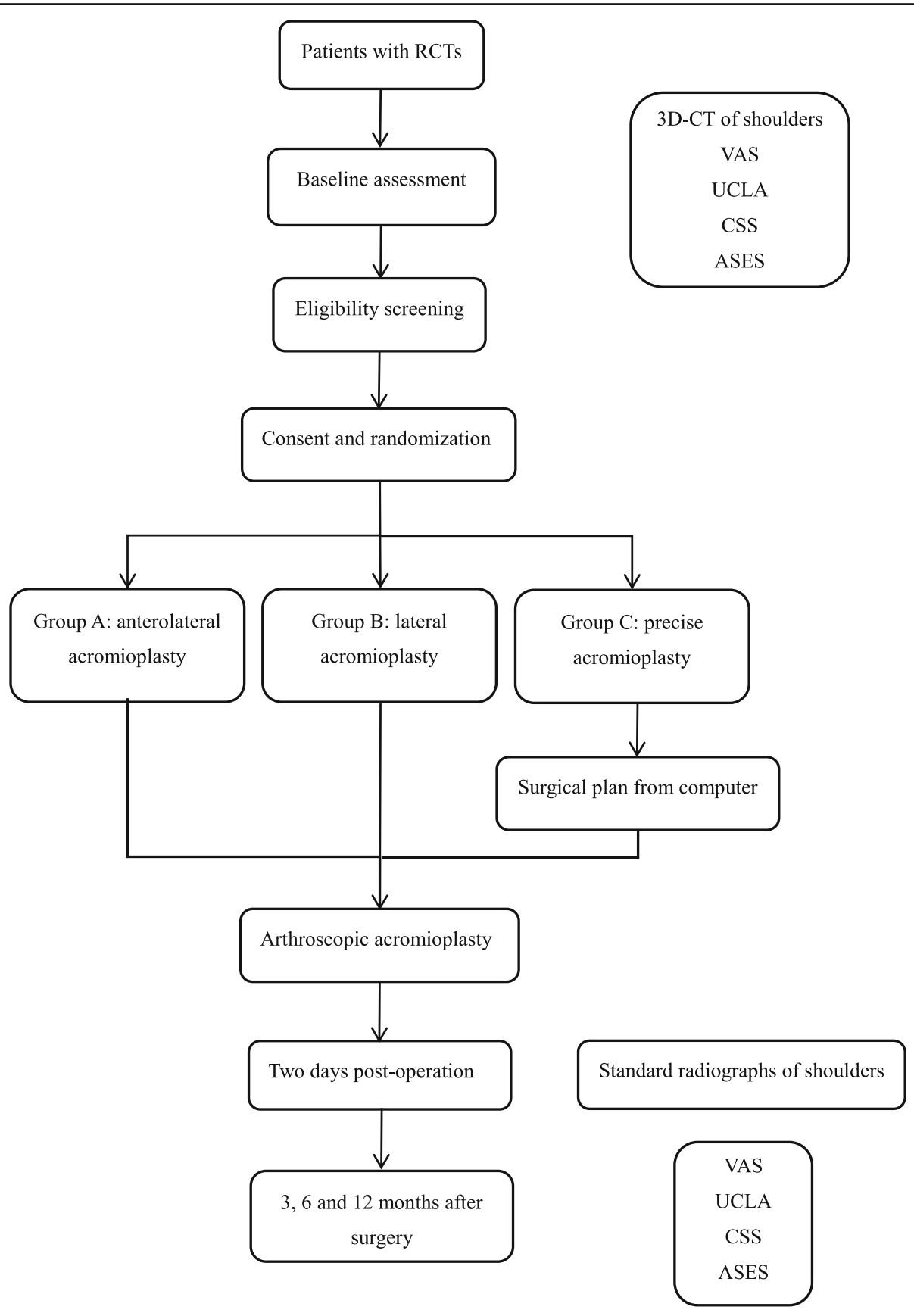

Fig. 1 Study flow diagram

operative assessment, and other researchers will blinded to the surgical decision making.

\section{Interventions}

All patients will receive a routine examination during admission, including routine blood and urine tests, as well as evaluation of general condition. Imaging examination, such as a 3D-CT of shoulder and MRI (if necessary), will be completed before enrollment. All participants will complete standard anteroposterior radiography of shoulders 2 days post-operation and follow-up will be continued at 3, 6 and 12 months after surgery. This trial will involve three types of acromioplasty: anterolateral, lateral or precise acromioplasty. One of these acromioplasties will be randomly performed along with arthroscopic RCR in each group.

\section{Surgical procedures}

One professional surgeon will make the surgical plan and conduct the arthroscopy in Sun Yat-sen Memorial Hospital. All participants will undergo general anaesthesia and be fixed 
in lateral decubitus. The surgery will begin with the establishment of a standard posterior portal and diagnostic arthroscopy. Then the arthroscopic RCR will be performed depending on the tear location and size. Later, three different acromioplasties will be conducted in groups A, B and C respectively according to a random allocation as previously described. The main processes of the acromioplasties are described below.

In group $\mathrm{A}$, after the undersurface of the acromioclavicular joint and the anterolateral margin of the acromion are freed from all soft tissue via a $5 \mathrm{~mm}$ shaver burr (Arthrex Inc., Naples, USA), the surgeon will gradually resect the anterolateral acromion from the anterolateral corner, perpendicular to the line from the anterior third lateral edge of the acromion to anterior acromioclavicular joint line margin. The anterolateral acromioplasty in group A will involve a width of approximately $10 \mathrm{~mm}$ (two shaver burr widths).

In group $\mathrm{B}$, after the undersurface and lateral margin of the acromion are freed from all soft tissue, the lateral acromioplasty will be started inferiorly at the middle lateral edge of the acromion, perpendicular to the line from the anterolateral to the posterolateral corner of the acromion. The lateral acromioplasty in group B will involve a width of approximately $10 \mathrm{~mm}$ (two shaver burr widths).

In group $C$, the pre-operative CSA $\left(\geq 33^{\circ}\right)$ will be measured on the $3 \mathrm{D}$ model. We will set $30^{\circ}$ for the CSA as the post-operative target degree. Second, the virtual acromioplasty will be simulated and the resected bone border will be measured upon the Mimics (Materialise, Leuven, Belgium), including the anterolateral corner of acromion (A), anteromedial resection margin (B), posterolateral resection margin $(C)$, and the distance of $A B$ and $\mathrm{AC}$. Third, the surgeon will identify and mark points $\mathrm{A}, \mathrm{B}$ and $\mathrm{C}$ on the patient's skin by measurement the distance of $\mathrm{AB}$ and $\mathrm{AC}$ according to the pre-operative planning above. Then, after the undersurface of the acromion are freed from all soft tissue, the surgeon will insert points $\mathrm{A}, \mathrm{B}$ and $\mathrm{C}$ with spinal needles perpendicularly from the skin to the subacromial space as line marks, a resection line will be made from $B$ to $C$ during arthroscopy. Lastly, the region of the acromion outside the resection line will be gradually resected by a $5 \mathrm{~mm}$ shaver burr (Arthrex Inc., Naples, FL, USA) during arthroscopy. The main procedure is shown schematically in Fig. 2.

\section{Outcome measures}

General information and baseline assessment will be collected during admission. The post-operative CSA will be measured 2 days post-operation. Follow-up will be maintained at 3, 6, and 12 months after surgery, including the visual analog scale (VAS) score, the University of
California at Los Angeles (UCLA) score, the Constant Shoulder Score (CSS) and the American Shoulder and Elbow Surgeon (ASES) Shoulder Assessment Form. All outcomes will be assessed by two researchers who are blinded to the recruitment and allocation.

\section{Primary outcome \\ Pre-operative CSA on 3D scapula}

The pre-operative CSA of patients will be measured on 3D-CT model before enrollment. First, Digital Imaging and Communications in Medicine (DICOM) data from CT scans of the shoulders will be used to reconstruct 3D scapulae by Mimics (Materialise, Leuven, Belgium). Then, the 3D model will be imported into Blender (Amsterdam, Netherlands) and aligned to the local coordinate system according to the same criteria as described by Suter et al. [28]. Lastly, the CSA, which is measured as the angle between a line connecting the inferior border of the glenoid with the most inferolateral point of the acromion and another connecting inferior and superior glenoid margin [12], will be measured on anterior view using Blender (Fig. 3a). Patients with a native CSA $\geq 33^{\circ}$ will be recruited as candidates.

\section{Post-operative CSA on X-ray}

The post-operative CSA of participants will be measured on standard anteroposterior radiography of the shoulder (Fig. 3b). The efficacy is reflected in the reduction of CSA after acromioplasty. The accuracy is defined as the percentage of patients whose postoperative CSA decreased from a large angle $\left(\geq 33^{\circ}\right)$ to a normal range $\left(30-33^{\circ}\right)$ via acromioplasty.

\section{Secondary outcome}

\section{Shoulder function scores}

Subjective pain will be ranked by the patients on the visual analog scale (VAS) score for pain, with 0 indicating no pain at all and 10 indicating the most severe pain the patient could imagine. The University of California at Los Angeles (UCLA) score, the Constant Shoulder Score (CSS) and the American Shoulder and Elbow Surgeon (ASES) Shoulder Assessment Form will be used for the functional assessment.

\section{Adverse event management}

In this study, the different interventions for participants who are willing to receive arthroscopic RCR are three diverse acromioplasties, which are relatively safe procedures during minimally invasive surgery. Adverse events are defined as any unexpected medical incidents which happen in participants and do not necessarily have a causality with the acromioplasty, including acromial fractures, detachment of deltoid origin, and either pain or swelling due to bone removal of acromion. 

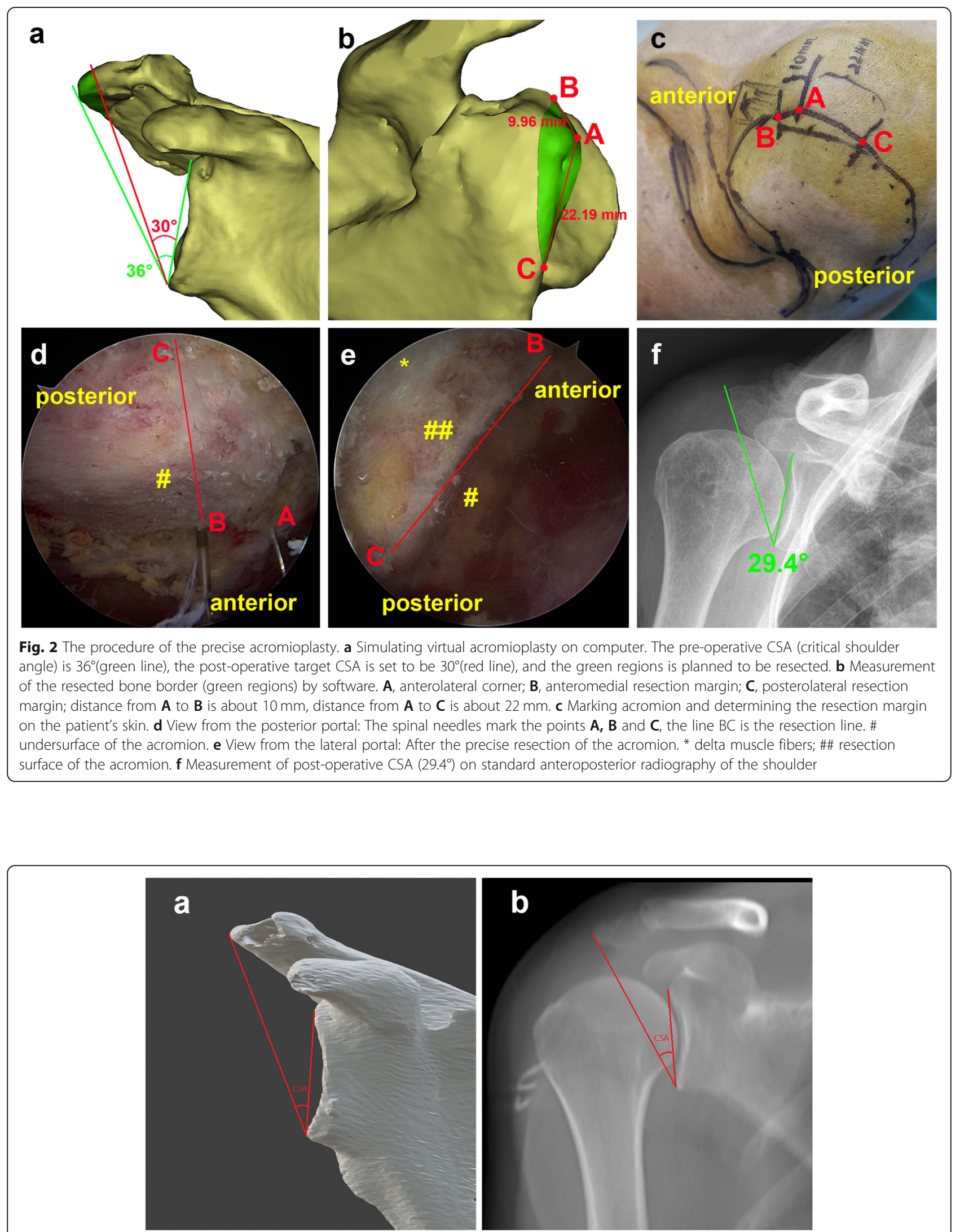

Fig. 3 The measurement of the critical shoulder angle (CSA) on 3D scapula (a) and X-ray (b) 
Participants will report any adverse events to doctors or study researchers as soon as they can. All adverse events will be documented and further medical treatment will be arranged if necessary. In addition, the participants will be closely followed up until conditions are resolved or their condition is stabilized.

\section{Data management}

Participants will be coded with a study number after informed consent, and all data referring to patients will be documented by this number, not by name. A case report form (CRF) will be used for participants to collect information including baseline information, radiographic outcomes, and follow-up. Data will be collected at baseline admission and 2 days post-operation, as well as 3, 6 and 12 months post-surgery. Data on adverse events and further medical treatments will also be collected. The recording of data is assigned to a data manager who is accountable for it, and other researchers will be restricted from accessing study data.

\section{Sample size calculation}

The primary outcome of this trial is the CSA and a power analysis was performed using PASS 15 (NCSS LLC, USA) to calculate the sample size. According to the results of Kaiser's study [26] and our preliminary study, the reduction of CSA was $2.6^{\circ} \pm 1.8^{\circ}$ (mean \pm standard deviation, $\mathrm{SD}$ ) in group $\mathrm{A}, 4.4^{\circ} \pm 1.5^{\circ}$ in group $\mathrm{B}$, and $4.8^{\circ} \pm 1.6^{\circ}$ in group $\mathrm{C}$ respectively. Based on a power of $90 \%$, and an alpha error of 0.05 , a sample size of 48 patients was determined. After taking into consideration a dropout rate of $20 \%$, a sample of 60 participants (20 participants per group) will be recruited.

\section{Statistical analysis}

The analysis of covariance will be conducted to compare the alteration of the CSA and shoulder function scores between randomized groups. The accuracy rates of different acromioplasties will be calculated, and $\chi^{2}$ test will be performed to evaluate the statistical differences between groups. The Spearman correlation coefficients will be used to assess the relationship between CSA and shoulder function scores. The intraclass correlation coefficient will be calculated to assess the consistency between researchers and between measurements of a single researcher. Statistical significance will be set at $P<0.05$. All analyses will be carried out using SPSS 19 (IBM Corp, USA) by two researchers blinded to the recruitment and collection.

\section{Discussion}

The critical shoulder angle (CSA), which combines the measurements of the lateral extension of the acromion and the inclination of the glenoid joint plane, was originally introduced by Moor et al. [12]. They documented that degenerative rotator cuff tears (RCTs) had significantly larger CSAs $\left(\geq 35^{\circ}\right)$ than asymptomatic shoulders without this pathology, and that a CSA smaller than $30^{\circ}$ is associated with glenohumeral osteoarthrtitis (OA). Gerber et al. confirmed these conclusions through a biomechanical analysis which demonstrated that a high CSA could induce supraspinatus (SSP) overload and that a low CSA increased the pressure of the humeral head on the glenoid [29-31]. To date, altering the CSA by arthroscopic acromioplasty is a common strategy. In 2017, Gerber et al. first demonstrated that the mean CSA was significantly corrected from $37.5^{\circ}$ pre-operatively to $33.9^{\circ}$ post-operatively through lateral acromioplasty in vivo [20]. Billaud et al. first reported in a clinical study that the average CSA decreased from $35.9^{\circ}$ to $33^{\circ}$ after anterolateral acromioplasty (anterior third of the acromion to be resected) [24]. Although arthroscopic acromioplasty is an effective procedure to decrease CSAs, questions remain regarding the necessity of acromioplasty performed alongside rotator cuff repair (RCR) has never ceased [32]. Several studies have found no difference in patient-reported outcome scores for patients who had arthroscopic RCR with or without acromioplasty [33-35]; besides, the reduction of the CSA does not improve functional results postoperatively [36, 37]. Conversely, some authors have demonstrated that large CSAs increase the risk of retearing after RCR [38-41]. Gerber et al. showed that abnormally large CSAs are associated either with higher re-tear rates or with inferior strength of abduction after reconstruction [20]. As most of these authors suggested, we recommend that if the CSA is greater than $33^{\circ}$, acromioplasty should be performed during arthroscopic RCR to reduce large CSAs to normal range (30-33 ) [21].

Although lateral acromioplasty has been documented to be the most effective way to decrease CSAs in vitro $[22,26]$, the post-operative angle is uncontrollable due to the insufficient or over-resection of the acromion. In other words, this is not always correct the large CSAs into the desired range of $30-33^{\circ}$. Additionally, lateral acromioplasty up to $10 \mathrm{~mm}$ has been considered a safe technique $[20,22,42]$, yet some authors reported that over-resection of the acromion has potential complications, such as detachment of the deltoid origin and acromial fractures [43-45]. In 2017, Karns et al. introduced the concept of the critical acromion point (CAP), which is the most inferolateral part of the acromion and the key point used to determine the degree of the CSA on true anteroposterior radiograph. They also proposed a virtual acromioplasty on three-dimensional (3D) scapulae models with resection of the acromion parallel to the glenoid face of $2.5 / 5 \mathrm{~mm}$ medial to the CAP [46]. This technique seemed to have the equivalent effect in 
altering the CSA while preserving more bone volume when compared with lateral acromioplasty. However, only $80 \%$ of specimens with a native CSA of $38^{\circ}$ or greater could be decreased to the acceptable range by this virtual acromioplasty; in addition, this technique has not been clinically realized so far.

To addresses the aforementioned deficiencies, we developed a precise acromioplasty technique, which is an individualized therapeutic strategy oriented by preoperative virtual acromioplasty on 3D-CT model and software analysis, for the purpose of reducing the CSA with greater efficacy and accuracy. Theoretically, we can clinically decrease large CSAs to any desired specific angle by this technique without cutting acromion not contributing to the CSA. Additionally, we will conduct a prospective randomized clinical trial to examine patients with RCTs and a CSA of $33^{\circ}$ or greater who undergo acromioplasty during arthroscopic RCR to evaluate the impact of different acromioplasties. This study will provide a new precision and individualized treatment to reduce CSA.

\section{Abbreviations}

ASES: American Shoulder and Elbow Surgeon Shoulder Assessment Form; BMI: Body mass index; CAP: Critical acromion point; CRF: Case report form; CSA: Critical shoulder angle; CSS: Constant Shoulder Score; DICOM: Digital Imaging and Communications in Medicine; MRI: Magnetic resonance imaging; OA: Osteoarthritis; RCR: Rotator cuff repair; RCTs: Rotator cuff tears; SLAP: Superior labrum from anterior to posterior lesion; SSP: Supraspinatus; 3D-CT: Three-dimensional computed tomography; UCLA: University of California at Los Angeles score; VAS: Visual analog scale score

\section{Acknowledgements}

We would like to acknowledge Lindsey Wilkerson and Dr. Phei Er Saw for proofreading this article.

\section{Authors' contributions}

RY conceived the study. YL wrote the paper. JH revised the manuscript. YT, $\mathrm{FL}, \mathrm{MY}$ and $\mathrm{CZ}$ contributed to the design of the study protocol. All authors have read and approved the manuscript.

\section{Funding}

This study was supported by the Natural Science Foundation of Guangdong Province (2018A030313780). The funding body did not take part in the design of the study or in the writing of the manuscript.

\section{Availability of data and materials}

The results of this trial will be made available upon request by the corresponding author.

\section{Ethics approval and consent to participate}

This research is approved by the Sun Yat-sen Memorial Hospital Medical Science Research Ethics Committee (2020-KY-015). Additionally, the research protocol has been registered in the Chinese Clinical Trial Registry (ChiCTR2000032343). All participants will be fully informed and will sign the written informed consent form in order to participate in the study.

\section{Consent for publication}

Not applicable.

\section{Competing interests}

The authors declare no competing interests.
Received: 2 June 2020 Accepted: 22 November 2020

Published online: 07 December 2020

\section{References}

1. Clayton RAE, Court-Brown CM. The epidemiology of musculoskeletal tendinous and ligamentous injuries. Injury. 2008;39(12):1338-44.

2. Jain NB, Higgins LD, Losina E, Collins J, Blazar PE, Katz JN. Epidemiology of musculoskeletal upper extremity ambulatory surgery in the United States, BMC Musculoskelet Disord. 2014;15:4

3. Ko J, Huang CC, Chen W, Chen C, Chen S, Wang C. Pathogenesis of partial tear of the rotator cuff: a clinical and pathologic study. J Shoulder Elb Surg. 2006;15(3):271-8.

4. Hashimoto T, Nobuhara K, Hamada T. Pathologic evidence of degeneration as a primary cause of rotator cuff tear. Clin Orthop Relat R. 2003;415:111-20

5. Matthews TJ, Hand GC, Rees JL, Athanasou NA, Carr AJ. Pathology of the torn rotator cuff tendon. Reduction in potential for repair as tear size increases. J Bone Joint Surg Br. 2006;88(4):489-95.

6. Balke M, Schmidt C, Dedy N, Banerjee M, Bouillon B, Liem D. Correlation of acromial morphology with impingement syndrome and rotator cuff tears. Acta Orthop. 2013;84(2):178-83.

7. Balke M, Liem D, Greshake O, Hoeher J, Bouillon B, Banerjee M. Differences in acromial morphology of shoulders in patients with degenerative and traumatic supraspinatus tendon tears. Knee Surg Sports Traumatol Arthrosc. 2016;24(7):2200-5.

8. Li X, Xu W, Hu N, Liang X, Huang W, Jiang D, Chen H. Relationship between acromial morphological variation and subacromial impingement: a threedimensional analysis. PLoS One. 2017;12(4):e176193.

9. Nyffeler RW, Werner CM, Sukthankar A, Schmid MR, Gerber C. Association of a large lateral extension of the acromion with rotator cuff tears. J Bone Joint Surg Am. 2006;88(4):800-5.

10. Andrade R, Correia AL, Nunes J, Xará-Leite F, Calvo E, Espregueira-Mendes J, Sevivas N. Is bony morphology and Morphometry associated with degenerative full-thickness rotator cuff tears? A systematic review and metaanalysis. Arthroscopy. 2019;35(12):3304-15.

11. Song JG, Yun SJ, Song YW, Lee SH. High performance of critical shoulder angle for diagnosing rotator cuff tears on radiographs. Knee Surg Sports Traumatol Arthrosc. 2019;27(1):289-98.

12. Moor BK, Bouaicha S, Rothenfluh DA, Sukthankar A, Gerber C. Is there an association between the individual anatomy of the scapula and the development of rotator cuff tears or osteoarthritis of the glenohumeral joint?: a radiological study of the critical shoulder angle. Bone Joint J. 2013; 95-B(7):935-41.

13. Rhee SM, Kim JY, Kim JY, Cho SJ, Kim JH, Rhee YG. The critical shoulder angle: can it be sufficient to reflect the shoulder joint without the humeral head? J Shoulder Elb Surg. 2019;28(4):731-41.

14. Moor BK, Röthlisberger M, Müller DA, Zumstein MA, Bouaicha S, Ehlinger M, Gerber C. Age, trauma and the critical shoulder angle accurately predict supraspinatus tendon tears. Orthop Traumatol Surg Res. 2014;100(5):489-94.

15. Mantell MT, Nelson R, Lowe JT, Endrizzi DP, Jawa A. Critical shoulder angle is associated with full-thickness rotator cuff tears in patients with glenohumeral osteoarthritis. J Shoulder Elb Surg. 2017;26(12):e376-81.

16. Shinagawa K, Hatta T, Yamamoto N, Kawakami J, Shiota Y, Mineta M, Itoi E. Critical shoulder angle in an east Asian population: correlation to the incidence of rotator cuff tear and glenohumeral osteoarthritis. J Shoulder Elb Surg. 2018;27(9):1602-6.

17. Blonna D, Giani A, Bellato E, Mattei L, Caló M, Rossi R, Castoldi F. Predominance of the critical shoulder angle in the pathogenesis of degenerative diseases of the shoulder. J Shoulder Elb Surg. 2016;25(8):1328-36.

18. Bjarnison AO, Sørensen TJ, Kallemose T, Barfod KW. The critical shoulder angle is associated with osteoarthritis in the shoulder but not rotator cuff tears: a retrospective case-control study. J Shoulder Elb Surg. 2017;26(12): 2097-102.

19. Spiegl UJ, Horan MP, Smith SW, Ho CP, Millett PJ. The critical shoulder angle is associated with rotator cuff tears and shoulder osteoarthritis and is better assessed with radiographs over MRI. Knee Surg Sports Traumatol Arthrosc. 2016;24(7):2244-51.

20. Gerber C, Catanzaro S, Betz M, Ernstbrunner L. Arthroscopic correction of the critical shoulder angle through lateral Acromioplasty: a safe adjunct to rotator cuff repair. Arthroscopy. 2018;34(3):771-80.

21. Tang Y, Hou J, Li Q, Li F, Zhang C, Li W, Yang R. The effectiveness of using the critical shoulder angle and acromion index for predicting rotator cuff 
tears: accurate diagnosis based on standard and nonstandard Anteroposterior radiographs. Arthroscopy. 2019;35(9):2553-61.

22. Katthagen JC, Marchetti DC, Tahal DS, Turnbull TL, Millett PJ. The effects of arthroscopic lateral Acromioplasty on the critical shoulder angle and the anterolateral deltoid origin: an anatomic cadaveric study. Arthroscopy. 2016; 32(4):569-75.

23. Altintas B, Kääb M, Greiner S. Arthroscopic lateral acromion resection (ALAR) optimizes rotator cuff tear relevant scapula parameters. Arch Orthop Traum Su. 2016;136(6):799-804

24. Billaud A, Cruz-Ferreira E, Pesquer L, Abadie P, Carlier Y, Flurin P. Does the critical shoulder angle decrease after anterior acromioplasty? Arch Orthop Traum Su. 2019;139(8):1125-32.

25. Gürpınar T, Polat B, Çarkçı E, Eren M, Polat AE, Öztürkmen Y. The Effect of Critical Shoulder Angle on Clinical Scores and Retear Risk After Rotator Cuff Tendon Repair at Short-term Follow Up. Sci Rep-Uk. 2019;9(1):12315.

26. Kaiser D, Bachmann E, Gerber C, Meyer DC. Influence of the site of acromioplasty on reduction of the critical shoulder angle (CSA) - an anatomical study. BMC Musculoskelet Disord. 2018;19(1):371.

27. Chan AW, Tetzlaff JM, Gotzsche PC, Altman DG, Mann H, Berlin JA, Dickersin K, Hrobjartsson A, Schulz KF, Parulekar WR, et al. SPIRIT 2013 explanation and elaboration: guidance for protocols of clinical trials. Ann Intern Med. 2013;346:e7586.

28. Suter T, Henninger HB, Zhang Y, Wylie JD, Tashjian RZ. Comparison of measurements of the glenopolar angle in 3D CT reconstructions of the scapula and 2D plain radiographic views. Bone Joint J. 2016;98-B(11):1510-6.

29. Viehöfer AF, Snedeker JG, Baumgartner D, Gerber C. Glenohumeral joint reaction forces increase with critical shoulder angles representative of osteoarthritis-a biomechanical analysis. J Orthop Res. 2016;34(6):1047-52.

30. Gerber C, Snedeker JG, Baumgartner D, Viehöfer AF. Supraspinatus tendon load during abduction is dependent on the size of the critical shoulder angle: a biomechanical analysis. J Orthop Res. 2014;32(7):952-7.

31. Viehöfer AF, Gerber C, Favre P, Bachmann E, Snedeker JG. A larger critical shoulder angle requires more rotator cuff activity to preserve joint stability. J Orthop Res. 2016;34(6):961-8.

32. McFarland EG, Matsen RFA, Sanchez-Sotelo J. Clinical faceoff: what is the role of Acromioplasty in the treatment of rotator cuff disease? Clin Orthop Relat R. 2018;476(9):1707-12.

33. Abrams GD, Gupta AK, Hussey KE, Tetteh ES, Karas V, Bach BR, Cole BJ, Romeo AA, Verma NN. Arthroscopic repair of full-thickness rotator cuff tears with and without Acromioplasty. Am J Sports Med. 2014;42(6):1296-303.

34. MacDonald P, McRae S, Leiter J, Mascarenhas R, Lapner P. Arthroscopic rotator cuff repair with and without Acromioplasty in the treatment of fullthickness rotator cuff tears. J Bone Joint Surg Am. 2011;93(21):1953-60.

35. Buss DD, Stern SH, Tervola N, McCarty LP, Giveans MR. Prevalence of Rotator Cuff Repairs with and Without Concomitant Subacromial Decompressions Has Not Changed. HSS J. 2018;14(2):123-7.

36. Lee M, Chen JY, Liow MHL, Chong HC, Chang P, Lie D. Critical shoulder angle and acromial index do not influence 24-month functional outcome after arthroscopic rotator cuff repair. Am J Sports Med. 2017;45(13):2989-94.

37. Kirsch JM, Nathani A, Robbins CB, Gagnier JJ, Bedi A, Miller BS. Is there an association between the "critical shoulder angle" and clinical outcome after rotator cuff repair? Orthop J Sports Med. 2017;5(4):1537111964.

38. Garcia GH, Liu JN, Degen RM, Johnson CC, Wong A, Dines DM, Gulotta LV, Dines JS. Higher critical shoulder angle increases the risk of retear after rotator cuff repair. J Shoulder Elb Surg. 2017;26(2):241-5.

39. Shi $X, X u Y$, Dai B, Li W, He Z. Effect of different geometrical structure of scapula on functional recovery after shoulder arthroscopy operation. J Orthop Surg Res. 2019;14(1):312.

40. Li H, Chen Y, Chen J, Hua Y, Chen S. Large critical shoulder angle has higher risk of tendon Retear after arthroscopic rotator cuff repair. Am J Sports Med. 2018;46(8):1892-900.

41. Scheiderer B, Imhoff FB, Johnson JD, Aglio J, Cote MP, Beitzel K, Imhoff AB, Arciero RA, Mazzocca AD, Morikawa D. Higher critical shoulder angle and acromion index are associated with increased Retear risk after isolated supraspinatus tendon repair at short-term follow up. Arthroscopy. 2018; 34(10):2748-54

42. Marchetti DC, Katthagen JC, Mikula JD, Montgomery SR, Tahal DS, Dahl KD, Turnbull TL, Millett PJ. Impact of arthroscopic lateral Acromioplasty on the mechanical and structural integrity of the lateral deltoid origin: a cadaveric study. Arthroscopy. 2017;33(3):511-7.
43. Matthews LS, Burkhead WZ, Gordon S, Racanelli J, Ruland L. Acromial fracture: a complication of arthroscopic subacromial decompression. J Shoulder Elb Surg. 1994;3(4):256-61.

44. Green A, Griggs S, Labrador D. Anterior acromial anatomy: relevance to arthroscopic acromioplasty. Arthroscopy. 2004;20(10):1050-4.

45. Torpey BM, Ikeda K, Weng M, van der Heeden D, Chao EY, McFarland EG, Wang $M$. The deltoid muscle origin. Histologic characteristics and effects of subacromial decompression. Am J Sports Med. 1998;26(3):379.

46. Karns MR, Jacxsens M, Uffmann WJ, Todd DC, Henninger HB, Burks RT. The critical acromial point: the anatomic location of the lateral acromion in the critical shoulder angle. J Shoulder Elb Surg. 2018;27(1):151-9.

\section{Publisher's Note}

Springer Nature remains neutral with regard to jurisdictional claims in published maps and institutional affiliations.

\section{Ready to submit your research? Choose BMC and benefit from:}

- fast, convenient online submission

- thorough peer review by experienced researchers in your field

- rapid publication on acceptance

- support for research data, including large and complex data types

- gold Open Access which fosters wider collaboration and increased citations

- maximum visibility for your research: over $100 \mathrm{M}$ website views per year

At $\mathrm{BMC}$, research is always in progress.

Learn more biomedcentral.com/submissions 